PROCEEDINGS OF THE

AMERICAN MATHEMATICAL SOCIETY

Volume 136, Number 8, August 2008, Pages 2683-2688

S 0002-9939(08)09195-8

Article electronically published on March 27, 2008

\title{
MODULAR FORMS OF HALF-INTEGRAL WEIGHT WITH FEW NON-VANISHING COEFFICIENTS MODULO $\ell$
}

\author{
D. CHOI
}

(Communicated by Ken Ono)

Abstract. Bruinier and Ono classified cusp forms of half-integral weight

$$
F(z):=\sum_{n=0}^{\infty} a(n) q^{n} \in S_{\lambda+\frac{1}{2}}\left(\Gamma_{0}(N), \chi\right) \cap \mathbb{Z}[[q]]
$$

whose Fourier coefficients are not well distributed for modulo odd primes $\ell$. Ahlgren and Boylan established bounds for the weight of such a cusp form and used these bounds to prove Newman's conjecture for the partition function for prime-power moduli. In this note, we give a simple proof of Ahlgren and Boylan's result on bounds of cusp forms of half-integral weight.

\section{INTRODUCTION AND RESULTS}

Much is known about the divisibility of the Fourier coefficients of modular forms of integral weight. When the weight is half-integral, the situation is not as clear. The distribution of the coefficients of modular forms of half-integral weight in congruence classes has been studied by, among others, Balog, Darmon, and Ono 3 , Ono and Skinner [8], 9], Brunier [4, Bruinier and Ono [5, and Ahlgren and Boylan [1, [2].

Let $S_{\frac{k}{2}}\left(\Gamma_{0}(N), \chi\right)$ be the space of cusp forms of weight $\frac{k}{2}$ on the congruence subgroup $\Gamma_{0}(N)$ with Dirichlet character $\chi$ (see Chapter 3 in [7 for the basic definitions of a cusp form). Recently Bruinier and Ono proved in [5] that $F(z):=$ $\sum_{n=0}^{\infty} a(n) q^{n} \in S_{\lambda+\frac{1}{2}}\left(\Gamma_{0}(N), \chi\right) \cap \mathbb{Z}[[q]]$ has a special form (see (1.1)) modulo an odd prime $\ell$ if $F(z)$ does not satisfy the following property: for every integer $r$

$$
\sharp\{1 \leq n \leq X \mid a(n) \equiv r \quad(\bmod \ell)\} \gg_{r, M}\left\{\begin{array}{lll}
\frac{\sqrt{X}}{\log X} & \text { if } r \not \equiv 0 & (\bmod \ell), \\
X & \text { if } r \equiv 0 & (\bmod \ell) .
\end{array}\right.
$$

Ahlgren and Boylan obtained the following bounds for the weights of cusp forms not satisfying the above property.

Theorem $1.1(2])$. Suppose that we have the following hypotheses:

(i) $\lambda \geq 2$ is an integer, $N$ is a positive integer with $4 \mid N$, and $\chi$ is a real Dirichlet character modulo $N$.

(ii) $\ell \geq 5$ is a prime such that $\ell \nmid N$.

Received by the editors January 12, 2007, and, in revised form, April 24, 2007.

2000 Mathematics Subject Classification. Primary 11F11, 11F33.

Key words and phrases. Modular forms, congruences.

(C)2008 American Mathematical Society Reverts to public domain 28 years from publication 
(iii) $F(z)$ is a cusp form of half-integral weight such that

$$
F(z)=\sum_{n=1}^{\infty} a(n) q^{n} \in S_{\lambda+\frac{1}{2}}\left(\Gamma_{0}(N), \chi\right) \cap \mathbb{Z}[[q]] .
$$

(iv) $F(z) \not \equiv 0(\bmod \ell)$, and there are finitely many square-free integers $n_{1}, n_{2}, \ldots$, $n_{t}$ such that

$$
F(z) \equiv \sum_{i=1}^{t} \sum_{m=1}^{\infty} a\left(n_{i} m^{2}\right) q^{n_{i} m^{2}} \quad(\bmod \ell) .
$$

Write $\lambda=\bar{\lambda}+i_{\lambda}(\ell-1)$ with $0 \leq \bar{\lambda} \leq \ell-2$. Then the following are true:

(1) If $\ell \nmid n_{i}$ for some $i$, then

$$
\bar{\lambda} \leq 2 i_{\lambda}+1
$$

(2) If $\ell \mid n_{i}$ for all $i$ and $\bar{\lambda} \leq \frac{\ell-3}{2}$, then

$$
\bar{\lambda} \leq 2 i_{\lambda}-\frac{\ell-1}{2} .
$$

(3) If $\ell \mid n_{i}$ for all $i$ and $\bar{\lambda} \geq \frac{\ell-1}{2}$, then

$$
\bar{\lambda} \leq 2 i_{\lambda}+\frac{\ell+3}{2} .
$$

In this note, we give an elementary proof of Theorem 1.1 (1). They used these bounds to prove Newman's conjecture [2] for the partition function for prime-power moduli. These bounds were proved in 2 2 by using Galois representations and Shimura-lifting. Here we obtain a simple proof of these bounds by arithmetic of filtrations of modular forms when $\ell \nmid n_{i}$ for some $i$.

\section{MODULAR FORMS MOD $\ell$}

Throughout this section, we fix an integer $N \geq 1$ and a prime $\ell \nmid N$. We will recall the definitions and results that will be needed for our proof. The theory of modular forms modulo $\ell$ was developed by Serre 10 and Swinnerton-Dyer [1] for forms of level 1 . We will recall the definitions and results that will be needed for the next section. For proofs when $N \geq 4$ and $\ell \nmid N$, see Gross $[6]$.

Denote by $M_{k}$ the space of modular forms of weight $k$ on $\Gamma_{1}(N)$ with integer coefficients. If $f \in M_{k}$, let $\tilde{f} \in(\mathbb{Z} / \ell \mathbb{Z})[[q]]$ be the (coefficient-wise) reduction of $f \bmod \ell$. We write $\widetilde{M_{k}}:=\left\{\tilde{f} \mid f \in M_{k}\right\}$ for the space of weight $k$ modular forms modulo $\ell$ with level $N$.

The filtration $\omega(\tilde{f})$ of a modular form $\tilde{f} \in \widetilde{M_{k}}$ is defined to be

$$
\omega(\tilde{f}):=\inf \left\{k^{\prime} \mid \tilde{f} \in \widetilde{M_{k^{\prime}}}\right\} .
$$

If $f \in M_{k}$, we sometimes write $\omega(f)$ instead of $\omega(\tilde{f})$ if no confusion can arise. Note that $\omega(\tilde{f})=-\infty$ if and only if $f \equiv 0(\bmod \ell)$. If $f \in M_{k}, g \in M_{k^{\prime}}$, and $f \equiv g \not \equiv 0$ $(\bmod \ell)$, then we must have $k \equiv k^{\prime}(\bmod \ell-1)$. From this it follows that $\omega(\tilde{f}) \equiv k$ $(\bmod \ell-1)$.

For an positive integer $m$, the operator $U_{m}$ acts on $q$-expansions by

$$
\sum_{n} a(n) q^{n} \mid U_{m}=\sum_{n} a(m n) q^{n} .
$$


Note that if $T_{\ell}$ denotes the $\ell$-th Hecke operator on $M_{k}$, then $f\left|T_{\ell} \equiv f\right| U_{\ell}(\bmod \ell)$, so $U_{\ell}$ acts on the spaces $\widetilde{M_{k}}$. We define the theta operator by its effect on Fourier expansions:

$$
\theta\left(\sum_{n} a(n) q^{n}\right)=\sum_{n} n a(n) q^{n} .
$$

We record some basic properties of the filtration of a modular form modulo $\ell$.

Lemma 2.1 ([6], [8], [11]). Let $f \in \widetilde{M_{k}}$. Write $k=\bar{k}+i_{k}(\ell-1)$ with $0 \leq \bar{k} \leq \ell-2$.

(1) If $f$ is not a constant modulo $\ell$, then $\omega(f)>0$.

(2) $\omega\left(f^{\ell}\right)=\ell \cdot \omega(f)$.

(3) $\bar{k} \leq \omega(f) \leq k$.

(4) $\omega(\theta f) \equiv k+\ell+1(\bmod \ell-1)$.

(5) Suppose that $f$ is not a constant modulo $\ell$. Then $\omega(\theta f) \leq \omega(f)+\ell+1$ with equality if and only if $\omega(f) \not \equiv 0(\bmod \ell)$.

\section{ProOF}

Proof of Theorem 1.1 (i). From the assumption take $n_{v} \in\left\{n_{1}, \ldots, n_{t}\right\}$ such that $\ell \nmid n_{v}$. Following the argument of Lemma 4.1 of [2], we can find primes $p_{v, 1}, \ldots, p_{v, s_{v}}$ greater than $\ell$, and a modular form $F_{v}(z) \in S_{\lambda+\frac{1}{2}}\left(\Gamma_{0}\left(N p_{v, 1}^{2} \cdots p_{v, s_{v}}^{2}, \chi\right)\right) \cap \mathbb{Z}[[q]]$ with the property that

$$
F_{v}(z) \equiv \sum_{\operatorname{gcd}\left(m, \prod_{j} p_{v, j}\right)=1} a\left(n_{v} m^{2}\right) q^{n_{v} m^{2}} \not \equiv 0 \quad(\bmod \ell) .
$$

We have

$$
F_{v}(z) \in S_{\lambda+\frac{1}{2}}\left(\Gamma_{0}\left(N n_{v} p_{v, 1}^{2} \cdots p_{v, s_{v}}^{2},\left(\frac{4 n_{v}}{\bullet}\right) \chi\right)\right)
$$

and

$$
F_{v}(z) \equiv \sum_{\operatorname{gcd}\left(m, \prod_{j} p_{v, j}\right)=1} a\left(n_{v} m^{2}\right) q^{m^{2}} \quad(\bmod \ell)
$$

(see Chapter 3 in [7]). Here, $\left(\frac{4 n_{v}}{\bullet}\right)$ denotes the Jacobi symbol. Note that

$$
\ell \nmid N n_{v} p_{v, 1}^{2} \cdots p_{v, s_{v}}^{2} .
$$

First, we consider the case that $\theta\left(F_{v}(z) \mid U_{n_{v}}\right) \equiv 0(\bmod \ell)$. Let

$$
T(z):=1+2 \sum_{n=1}^{\infty} q^{n^{2}} \in M_{\frac{1}{2}}\left(\Gamma_{0}(4)\right) .
$$

In this case, $F_{v}(z) \mid U_{n_{v}}$ has the form

$$
F_{v}(z) \mid U_{n_{v}} \equiv \sum_{\operatorname{gcd}\left(m, \prod_{j} p_{v, j}\right)=1} a\left(n_{v} \ell^{2} m^{2}\right) q^{\ell^{2} m^{2}} \quad(\bmod \ell) .
$$

This implies that $\left(F_{v}(z) \mid U_{n_{v}} U_{\ell^{2}}\right)^{\ell^{2}} \equiv F_{v}(z) \mid U_{n_{v}}(\bmod \ell)$. Thus, we have

$$
2 i_{\lambda}+1 \geq \ell+2 \geq \bar{\lambda}
$$

since from Lemma 2.1 (2) and (3)

$$
\lambda+\frac{1+\ell^{2}}{2} \geq \omega\left(F_{v}(z) \mid U_{n_{v}} \cdot T(z)^{\ell^{2}}\right)=\omega\left(F_{v}(z) \mid U_{n_{v}} U_{\ell^{2}} \cdot T(z)\right)^{\ell^{2}} \geq \ell^{2} .
$$


From now we assume that $\theta\left(F_{v}(z) \mid U_{n_{v}}\right) \not \equiv 0(\bmod \ell)$. Let

$$
G(z):=F_{v}(z) \mid U_{n_{v}} \cdot T(z)^{\ell} .
$$

Since $4 \mid N$,

Then we have

$$
G(z) \in S_{\lambda+\frac{\ell+1}{2}}\left(\Gamma_{1}\left(N n_{v} p_{v, 1}^{2} \cdots p_{v, s_{v}}^{2}\right)\right)
$$

$$
\begin{aligned}
\theta^{\frac{\ell+1}{2}} G(z) & \equiv \theta^{\frac{\ell+1}{2}}\left(F_{v}(z) \mid U_{n_{v}}\right) T(z)^{\ell} \\
& \equiv \sum_{m=1}^{\infty} \theta^{\frac{\ell+1}{2}}\left(a\left(n_{i} m^{2}\right) q^{m^{2}}\right) T(z)^{\ell} \equiv \sum_{m=1}^{\infty}\left(a\left(n_{v} m^{2}\right) m^{2 \cdot \frac{\ell+1}{2}} q^{m^{2}}\right) T(z)^{\ell} \\
& \equiv \theta\left(F_{v}(z) \mid U_{n_{v}}\right) T(z)^{\ell} \equiv \theta G(z) \quad(\bmod \ell) .
\end{aligned}
$$

Let $\omega(\theta G(z)):=(m \ell+r)(\ell-1)+\bar{\lambda}+\frac{\ell+1}{2}+\ell+1=(m \ell+r+1-m) \ell-r+\bar{\lambda}+\frac{\ell+3}{2}$ by Lemma 2.1 (3), (4) and (5), where $m$ and $r$ are integers such that $m \ell+r \leq i_{\lambda}$ and $0 \leq r \leq \ell-1$. Let

$$
\beta(r):=\left\{\begin{array}{cc}
-r & \text { if } m \geq 0 \\
(m \ell+r+1-m) \ell-r & \text { if } m<0 .
\end{array}\right.
$$

Then $\beta(r) \leq 1$. If $m<0$, then $m=-1$ and $r=\ell-1$ or $\ell-2$ since $\omega(\theta G(z))=$ $\beta(r)+\bar{\lambda}+\frac{\ell+3}{2}>0$. If $m \geq 0$ and $-r+\bar{\lambda}+\frac{\ell+3}{2} \leq 0$, then

$$
2 i_{\lambda} \geq 2 r \geq \ell+3>\bar{\lambda}
$$

Thus, we may assume that

$$
0<\beta(r)+\bar{\lambda}+\frac{\ell+3}{2}<\ell+\frac{\ell+3}{2} .
$$

Note that

$$
\omega(\theta G(z))-\left(\beta(r)+\bar{\lambda}+\frac{\ell+3}{2}\right) \geq 0 \text { and } \omega(\theta G(z)) \equiv \beta(r)+\bar{\lambda}+\frac{\ell+3}{2} \quad(\bmod \ell) .
$$

If $\beta(r)+\bar{\lambda}+\frac{\ell+3}{2}<\frac{\ell+3}{2}$ or $\ell<\beta(r)+\bar{\lambda}+\frac{\ell+3}{2}$, then

$$
\omega\left(\theta^{\frac{\ell+1}{2}} G(z)\right)=\omega(\theta G(z))+(\ell+1) \frac{\ell-1}{2}>\omega(\theta G(z))
$$

by Lemma 2.1 (5). This contradicts (3.1). Thus, we have

$$
\frac{\ell+3}{2} \leq \beta(r)+\bar{\lambda}+\frac{\ell+3}{2} \leq \ell
$$

This implies that if $m=-1$ and $r=\ell-2$, then $\bar{\lambda}=\ell-2$. Suppose that $m=-1$ and $r=\ell-2$. Then, $\omega(G(z)) \equiv 0(\bmod \ell)$ by Lemma 2.1 (5) since $\omega(\theta G(z))<\ell+1$. So, if $\omega(G(z)):=\left(i_{\lambda}-t\right)(\ell-1)+\frac{\ell+1}{2}+\ell-2$ for some nonnegative integer $t \leq i_{\lambda}+1$, then

We have

$$
\omega(G(z)) \equiv-i_{\lambda}+t+\frac{\ell+1}{2}-2 \equiv 0 \quad(\bmod \ell) .
$$

$$
2 i_{\lambda} \geq 2\left(i_{\lambda}-t\right) \geq \ell-3=\bar{\lambda}-1 .
$$

Thus, from now on, we assume that $r=\ell-1$ if $m=-1$.

Now we consider the value $\omega\left(\theta^{\frac{\ell+1}{2}} G(z)\right)$. Combining Lemma 2.1 (3) and (4), we obtain

$$
\begin{aligned}
& \omega\left(\theta^{\ell-\left(\beta(r)+\bar{\lambda}+\frac{\ell+3}{2}\right)+2} G(z)\right) \\
& =\omega(\theta G(z))+\left(\ell-\left(\beta(r)+\bar{\lambda}+\frac{\ell+3}{2}\right)+1\right)(\ell+1)-\alpha(\ell-1) \\
& =\left(m \ell+r-\beta(r)+\frac{\ell+3}{2}-m-\bar{\lambda}^{2}-\alpha\right) \ell+1+\alpha-r-\beta(r),
\end{aligned}
$$


where $\alpha$ is a nonnegative integer. Since $\left(\ell-\left(\beta(r)+\bar{\lambda}+\frac{\ell+3}{2}\right)+2\right)+(\beta(r)+\bar{\lambda})=$ $\frac{\ell+1}{2}$, Lemma 2.1 (5) implies that

$$
\omega\left(\theta^{\ell-\left(\beta(r)+\bar{\lambda}+\frac{\ell+3}{2}\right)+2} G(z)\right)+(\beta(r)+\bar{\lambda})(\ell+1) \geq \omega\left(\theta^{\frac{\ell+1}{2}} G(z)\right)=\omega(\theta G(z)) .
$$

Thus, from (3.3) we have

$$
\frac{\ell+1}{2} \geq \alpha
$$

Note that $\omega\left(\theta^{\ell-\left(\beta(r)+\bar{\lambda}+\frac{\ell+3}{2}\right)+2} G(z)\right) \equiv 1+\alpha(\bmod \ell)$. Since by (3.2)

$$
0<1+\alpha+\beta(r)+\bar{\lambda} \leq \ell
$$

Lemma 2.1 (5) implies that

$$
\omega(\theta G(z))=\omega\left(\theta^{\frac{\ell+1}{2}} G(z)\right)=\omega\left(\theta^{\ell-\left(\beta(r)+\bar{\lambda}+\frac{\ell+3}{2}\right)+2} G(z)\right)+(\beta(r)+\bar{\lambda})(\ell+1) .
$$

Thus, we have

$$
\alpha=\frac{\ell+1}{2}
$$

Note that $\omega\left(\theta^{\ell-\left(\beta(r)+\bar{\lambda}+\frac{\ell+3}{2}\right)+2} G(z)\right)>0$ by Lemma 2.1 (11), and that $m=-1$ and $r=\ell-1$ if $m<0$. Thus, by using (3.3) and (3.4), we obtain

$$
\begin{array}{cl}
m \ell+2 r-m-\bar{\lambda}+1 \geq 0 & \text { if } m \geq 0, \\
\bar{\lambda} \leq-1 & \text { if } m<0 \text { and } r=\ell-1 .
\end{array}
$$

We complete the proof by the following inequalities: for $m \geq 0$,

$$
2 i_{\lambda} \geq 2(m \ell+r) \geq m \ell+2 r \geq m+\bar{\lambda}-1 \geq \bar{\lambda}-1 \text {. }
$$

\section{ACKNOWLEDGEMENTS}

We are very grateful to S. Ahlgren for helpful comments. We also thank the referee for useful comments.

\section{REFERENCES}

[1] S. Ahlgren and M. Boylan, Arithmetic properties of the partition function. Invent. Math. 153(3):487-502, 2003. MR2000466 (2004e:11115)

[2] S. Ahlgren and M. Boylan, Coefficients of half-integral weight modular forms modulo $l^{j}$. Math. Ann. 331(1):219-239, 2005. MR:2107445 (2005k:11091)

[3] Antal Balog, Henri Darmon and Ken Ono, Congruence for Fourier coefficients of half-integral weight modular forms and special values of L-functions. (English summary) Analytic number theory, Vol. 1 (Allerton Park, IL, 1995), 105-128, Progr. Math., 138, Birkhäuser Boston, Boston, MA, 1996. MR 1399333 (97e:11056)

[4] Jan Hendrik Bruinier, Non-vanishing modulo $\ell$ of Fourier coefficients of half-integral weight modular forms. Duke Math. J. 98(3):595-611, 1999. MR1695803(2000d:11061)

[5] J. H. Bruinier and K. Ono, Coefficients of half-integral weight modular forms, J. Number Theory 99(1): 164-179, 2003. MR1957250 (2004b:11056)

[6] Benedict H. Gross, A tameness criterion for Galois representations associated to modular forms $(\bmod p)$. Duke Math. J. 61(2):445-517, 1990. MR.1074305 (91i:11060)

[7] K. Ono, The web of modularity: Arithmetic of the coefficients of modular forms and $q$ series, CBMS Regional Conf. Series in Math., vol. 102, Amer. Math. Soc., 2004. MR2020489 (2005c:11053)

[8] Ken Ono, Christopher Skinner, Fourier coefficients of half-integral weight modular forms modulo l, Ann. of Math. (2), 147(2):453-470, 1998. MR1626761(99f:11059a) 
[9] Ken Ono, Christopher Skinner, Corrigendum: "Fourier coefficients of half-integral weight modular forms modulo l", Ann. of Math. (2), 148(1):361, 1998. MR.1652912 (99f:11059b)

[10] Jean-Pierre Serre, Formes modulaires et fonctions zeta p-adiques (French). Modular functions of one variable, III (Proc. Internat. Summer School, Univ. Antwerp, 1972), pp. 191-268. Lecture Notes in Math., vol. 350, Springer, Berlin, 1973. MR0404145 (53:7949a)

[11] H. P. F. Swinnerton-Dyer, On l-adic representations and congruences for coefficients of modular forms. Modular functions of one variable, III (Proc. Internat. Summer School, Univ. Antwerp, 1972), pp. 1-55. Lecture Notes in Math., vol. 350, Springer, Berlin, 1973. MR0406931(53:10717a)

School of Mathematics, KIAS, 207-43 Cheongnyangni 2-Dong 130-722, Korea

E-mail address: choija@postech.ac.kr 